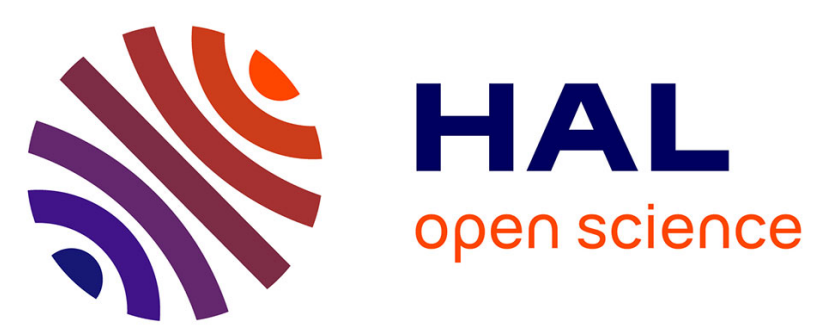

\title{
Structure des couches de niobium préparées par pulvérisation triode. Existence d'une phase cubique à faces centrées
}

C. Sella, L. Tertian, J. Deschamps

\section{- To cite this version:}

C. Sella, L. Tertian, J. Deschamps. Structure des couches de niobium préparées par pulvérisation triode. Existence d'une phase cubique à faces centrées. Revue de Physique Appliquée, 1970, 5 (3), pp.415-422. 10.1051/rphysap:0197000503041500 . jpa-00243410

\section{HAL Id: jpa-00243410 https://hal.science/jpa-00243410}

Submitted on 1 Jan 1970

HAL is a multi-disciplinary open access archive for the deposit and dissemination of scientific research documents, whether they are published or not. The documents may come from teaching and research institutions in France or abroad, or from public or private research centers.
L'archive ouverte pluridisciplinaire HAL, est destinée au dépôt et à la diffusion de documents scientifiques de niveau recherche, publiés ou non, émanant des établissements d'enseignement et de recherche français ou étrangers, des laboratoires publics ou privés. 


\title{
STRUCTURE DES COUCHES DE NIOBIUM PRÉPARÉES PAR PULVÉRISATION TRIODE EXISTENCE D’UNE PHASE CUBIQUE A FACES CENTRÉES
}

\author{
C. SELLA, L. TERTIAN et J. DESCHAMPS \\ Laboratoire de Rayons X, C. N. R. S., 92, Bellevue
}

\begin{abstract}
Résumé. - Par pulvérisation cathodique, le niobium, normalement cubique centré $(a=3,29 \AA)$ donne sur $\mathrm{NaCl}$ des couches amorphes sur support froid, désorientées au-dessous de $100^{\circ}$, épitaxiques entre 100 et $400^{\circ}$ avec les orientations $(110)_{\mathrm{Nb}} / /(100)_{\mathrm{NaCl}},(111)_{\mathrm{Nb}} / /(100)_{\mathrm{NaCl}}$ et $(100)_{\mathrm{Nb}} / /$ $(100)_{\mathrm{NaCl}}$.

Dans les premiers stades de la pulvérisation, on peut observer une structure anormale cubique à faces centrées $(a=4,42 \AA)$, souvent orientée suivant $(100)$ c. f. c.//(100) $)_{\mathrm{NaCl}}$, qui résulte d'une interaction du métal avec certains gaz résiduels présents dans l'enceinte ou à la surface du substrat. Sa formation peut être évitée en dégazant soigneusement le substrat et en purifiant l'atmosphère résiduelle par une pulvérisation préalable prolongée destinée à fixer tous les gaz actifs.

On tente de préciser la nature de cette phase en étudiant son évolution, dans le vide résiduel d'un diffractographe, jusqu'à environ $800^{\circ} \mathrm{C}$.
\end{abstract}

\begin{abstract}
Sputtering of normally b. c. c. Niobium $(a=3.29 \AA)$ on Sodium Chloride gives amorphous films on cold substrate, polycrystalline disoriented films below $100^{\circ} \mathrm{C}$, and epitaxial layers, with ${ }^{(110)} \mathrm{Nb} / /^{(100)} \mathrm{NaCl},{ }^{(111)} \mathrm{Nb} / /^{(100)} \mathrm{NaCl}$ and ${ }^{(10)} \mathrm{Nb} / /^{(100)} \mathrm{NaCl}$ orientations, between 100 and $400{ }^{\circ} \mathrm{C}$.

During the first stage of sputtering an abnormal f. c. c. structure $(a=4.42 \AA)$ often oriented with $(100) / /(100) \mathrm{NaCl}$, has been observed. It results from the interaction between the metal and some residual gas present in the sputtering chamber or on the substrate surface. Its formation can be avoided by a careful outgasing of the substrate or a purification of the residual atmosphere by a long preliminary sputtering in order to fix all active gas.

An attempt to specify the nature of this abnormal phase has been made by studying its evolution in the diffractograph residual atmosphere up to about $800^{\circ} \mathrm{C}$.
\end{abstract}

Introduction. - Les couches minces de Niobium présentent un grand intérêt dans la mesure où elles peuvent être préparées avec de bonnes propriétés supraconductrices. Elles conduisent alors à de nombreuses applications (cryotrons, mémoires de machines à calculer, lignes de transport de courant électrique, générateurs supraconducteurs, réalisation de champs magnétiques intenses, cavités hyperfréquence, etc.).

Le niobium comme les métaux dits réfractaires ( Ta, Mo, W, Zr) ne peut être évaporé par les méthodes d'évaporation thermique classiques. L'évaporation par bombardement électronique avec un canon très puissant est possible mais les propriétés supraconductrices des couches ainsi préparées ne sont pas excellentes même si l'évaporation est effectuée sous ultravide.

La technique de pulvérisation cathodique semble actuellement la meilleure méthode pour obtenir des couches minces de niobium présentant de bonnes propriétés supraconductrices.

Dans le présent travail nous avons étudié la structure des couches préparées par pulvérisation triode en fonction des conditions de pulvérisation et en particulier en fonction de la composition de l'atmosphère résiduelle de l'enceinte.
Dispositif de pulvérisation. - Le dispositif utilisé est celui du système à configuration triode. La cible est une plaque de niobium pur disposée verticalement à $5 \mathrm{~cm}$ du substrat.

Dans l'enceinte, préalablement vidée jusqu'à environ $10^{-6}$ torr, on admet de l'argon très pur et on forme un plasma stable par l'intermédiaire d'un faisceau d'électrons émis par un filament de tungstène chauffé, placé à la partie inférieure de l'enceinte. L'anode, portée à une centaine de volts, attire les électrons, et une bobine magnétique permet de concentrer le plasma dans une zone convenable. On obtient ainsi un régime stable d'ionisation avec une pression d'argon supérieure à $10^{-4}$ torr. On accélère alors les ions argon du plasma sur la cible en portant celle-ci à une tension négative de $1000 \mathrm{~V}$ par rapport au substrat. La cible est pulvérisée et l'épaisseur du dépôt croît régulièrement. Avec les conditions de travail choisies nous avons mesuré une vitesse de croissance de l'ordre de $150 \AA$ / minute.

Sur la figure 1 on peut voir la cible et le dispositif de chauffage du substrat. Un écran mobile, placé devant le four, permet d'une part, de faire varier l'épaisseur des couches et d'autre part, de faire une pulvérisation préalable de la cible avant la condensa- 


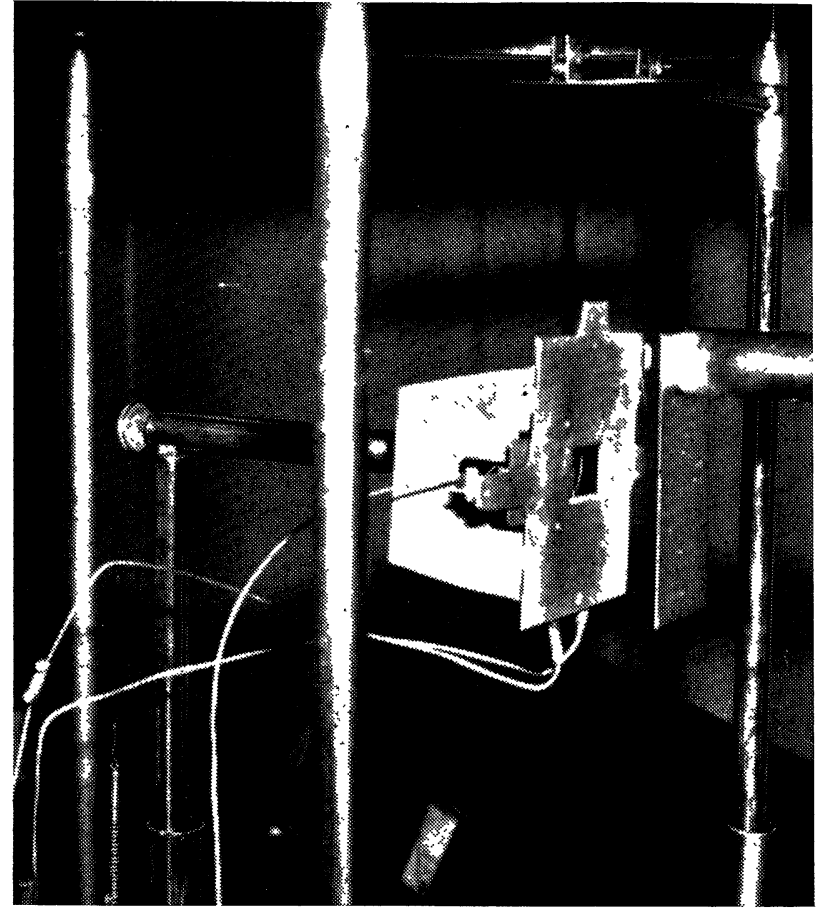

Fig. 1. - Détail du dispositif de pulvérisation triode : anode, cible, dispositif de chauffage du substrat et écran mobile.

tion. On obtient par cette méthode, des couches en principe très pures, très adhérentes, qui se forment à partir de germes nombreux et sont, de ce fait, continues, pour des épaisseurs moyennes relativement faibles.

Morphologie et structure des couches condensées sur NaCl. - Nous avons examiné au microscope électronique la morphologie des dépôts recueillis pendant quelques minutes sur des faces de clivage de $\mathrm{NaCl}$ portées à différentes températures, entre 20 et $400^{\circ} \mathrm{C}$. et étudié leur structure et leur orientation par diffraction électronique.

1. Couches DE NIOBIUM CUBiQue CENTRÉ. - Dans une première série d'expériences nous avons étudié essentiellement l'influence de la température du substrat. La pression d'argon était de 7 à $8 \times 10^{-4}$ torr pendant la pulvérisation et presque tous les diagrammes ont pu être interprétés à partir de la maille cubique centrée du niobium d'arête $a=3,29 \AA$.

La figure 2 montre l'aspect général de ces couches au microscope électronique : elles sont très homogènes et formées de grains très petits (100 à $500 \AA$ suivant la température du substrat).

Les dépôts obtenus à froid sont généralement amorphes ou très mal cristallisés. Au-dessus de $50^{\circ}$ et jusque vers $100^{\circ}$ ils sont cristallisés mais ne présentent pas d'orientation préférentielle notable. Le diagramme est celui du Niobium c. c. polycristallin (Fig. 3).

$\mathrm{Au}$-dessus de $100^{\circ}$ et surtout vers $200-300^{\circ}$, on observe presque toujours une ou plusieurs orien-
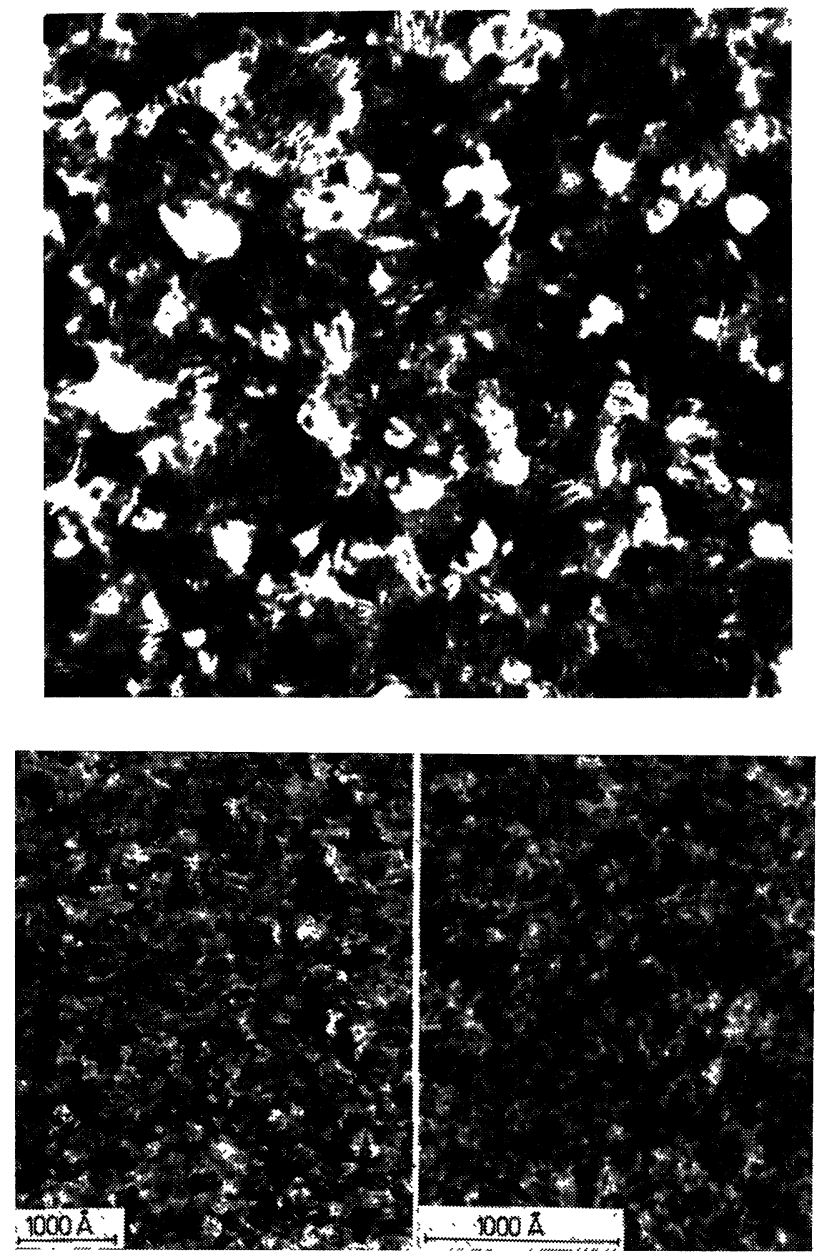

(a)

(b)

Fig. 2. - Aspect général des couches au microscope électronique. Examen direct par transmission.

a) Couche c. c. b) Couche c. f. c.

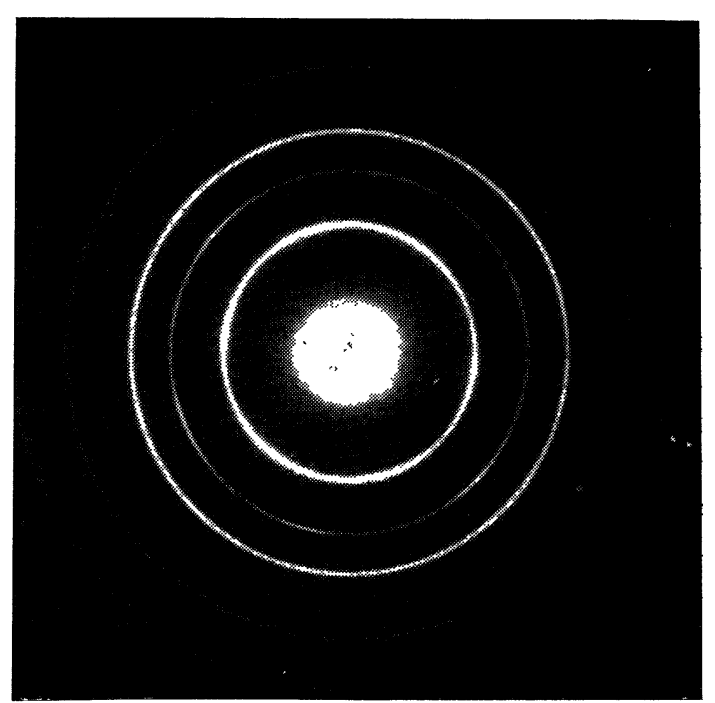

FIG. 3. - Nb c. c. polycristallin.

tations préférentielles des cristallites. La plus fréquente correspond au plan $(001)_{\mathrm{Nb}} / /(001)_{\mathrm{NaCl}}$ avec $(100)_{\mathrm{Nb}} / /(110)_{\mathrm{NaCl}}$. 
Plus rarement on a pu observer un autre type de diagramme (Fig. 4) dont l'interprétation est

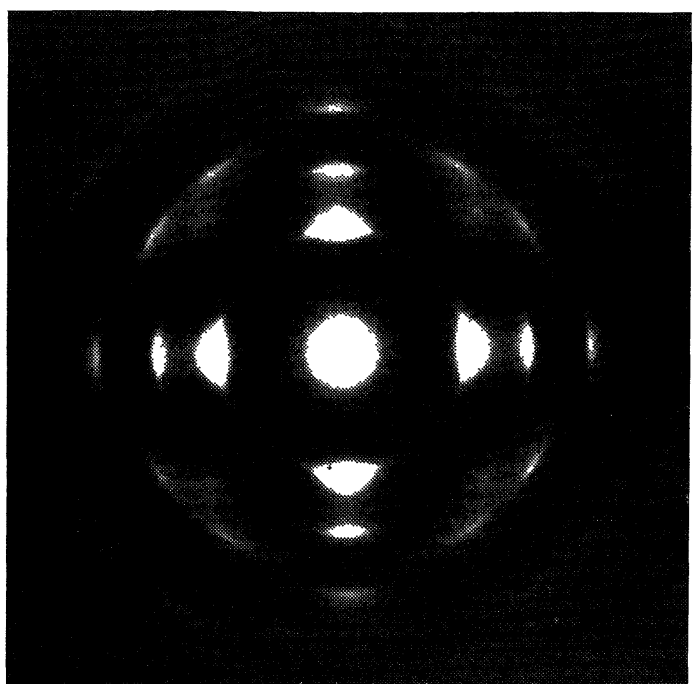

FIG. $4 .-\mathrm{Nb}$ c. c. : orientation préférentielle $(110)_{\mathrm{Nb}} / /(001)_{\mathrm{NaCl}}$.

représentée sur le schéma figure 5 : on a un plan $(110)_{\mathrm{Nb}} / /(001)_{\mathrm{NaCl}}$ avec $(100)_{\mathrm{Nb}} / /$ soit à $(100)_{\mathrm{NaCl}}$ soit à $(010)_{\mathrm{NaCl}}$, ce qui donne deux familles de taches (ou d'arcs) symétriques par rapport à la première bissectrice.

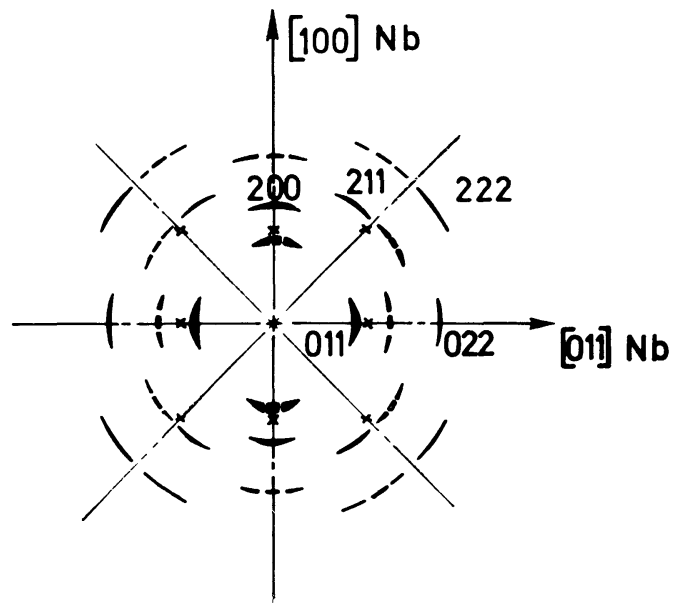

FIG. 5. - Indiçage du diagramme (4). Les croix correspondent à des taches floues du diagramme (taches (hko) de la forme c. f. c.

Enfin très souvent le diagramme (Fig. 6) comporte, en plus des taches (hko) correspondant à l'orientation (001), des taches supplémentaires, sur l'anneau (110) en particulier, qui peuvent s'expliquer si on a $(111)_{\mathrm{Nb}} / /(001)_{\mathrm{NaCl}}$ dans deux azimuts décalés de $90^{\circ}$.

2. Couches ayant UNE STRucture CUbiQue a FACES CENTRÉES. - En travaillant sensiblement dans les mêmes conditions de pression, de chauffage filament et de tension anode et cible pour préciser l'importance de certains facteurs responsables de l'orientation (température du substrat, épaisseur des couches, par ex.) on obtint dans certains cas des préparations

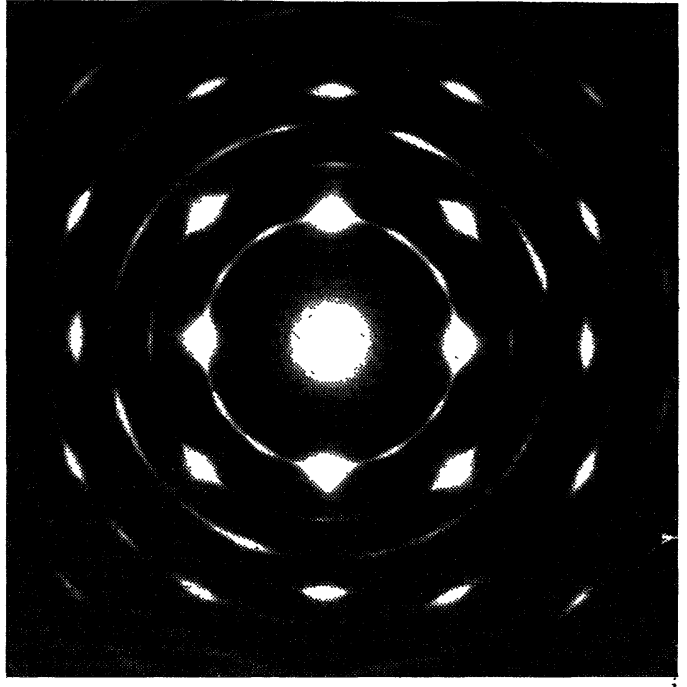

FIG. 6. - Nb c. c. Orientations préférentielles $(001)_{\mathrm{Nb}} / /(001)_{\mathrm{NaCl}}$ et $(111)_{\mathrm{Nb}} / /(001)_{\mathrm{NaCl}}$ dans 2 azimuts à $90^{\circ}$.

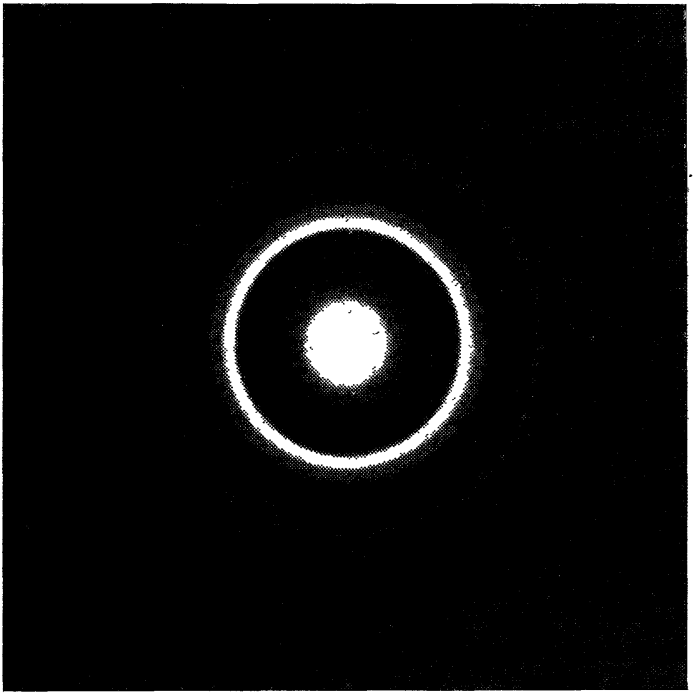

Fig. 7. - Forme c. f. c. non orientée.

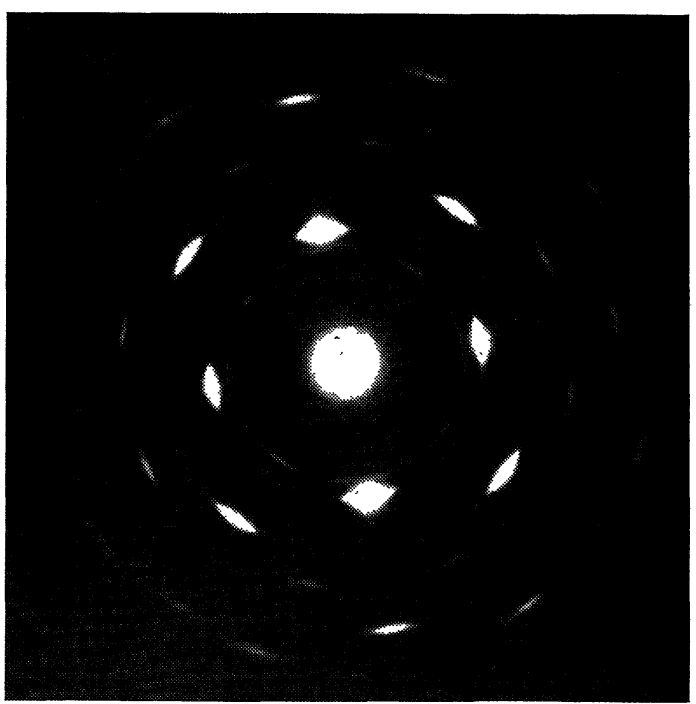

FIG. 8. — Forme c. f. c. orientation préférentielle $(001) / /(001)_{\mathrm{NaCl}}$. 
qui n'avaient pas la structure habituelle du niobium cubique centré et dont les diagrammes, souvent très bien orientés, s'interprétaient parfaitement à partir d'une maille c. f. c. de paramètre $a$ voisin de $4,42 \AA$ et maclée suivant les plans $\{111\}$.

Les figures 7,8 et 9 montrent, respectivement, le diagramme d'une couche recueillie sur $\mathrm{NaCl}$ à $100^{\circ}$ environ, sur lequel on reconnaît bien les anneaux d'un corps polycristallin c. f. c., la même structure avec l'orientation préférentielle (001) et des macles de croissance, et enfin un diagramme particulièrement bien orienté obtenu en faisant le dépôt sur $\mathrm{NaCl}$ à $300^{\circ}$.

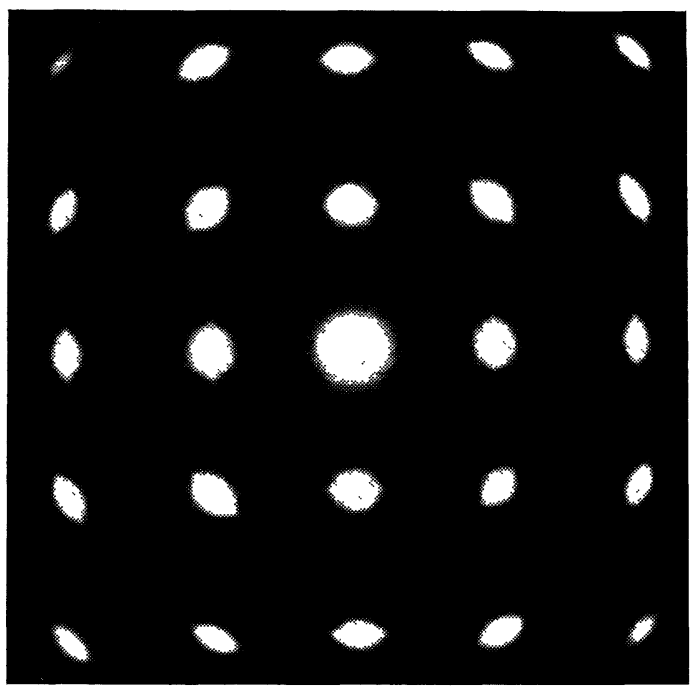

FIG. 9. - Forme c. f. c. très bien orientée (001)//substrat $\left(300^{\circ}\right)$

Origine de la forme cubique à faces centrées. L'existence de cette forme c. f. c. n'est pas particulière au niobium. C. Sella [1], par pulvérisation cathodique et d'autres auteurs dont M. Gillet [2], par bombardement électronique, ont obtenu également avec le tungstène, normalement c. c., une forme c. f. c. K. L. Chopra et al. [3] signalent un phénomène analogue en pulvérisant sur différents substrats des métaux normalement c. c. ou hexagonaux comme le tantale, le tungstène, le rhénium, le hafnium et le zirconium. Ils retrouvent la structure normale en faisant un recuit vers $700^{\circ}$ et pensent qu'il s'agit d'une modification allotropique métastable.

T. E. Hutchinson et K. H. Olsen [4] obtiennent une forme c. f. c. en condensant sur un monocristal de $\mathrm{MgO}$ des films de niobium évaporé par bombardement électronique et font intervenir une réaction chimique à la surface du substrat avec formation d'un sous-oxyde intermédiaire.

Par ailleurs, J. Sosniak [5] obtient des dépôts de niobium ayant sensiblement les propriétés du métal massif par pulvérisation diode sur des substrats chauffés à température assez élevée $\left(700^{\circ}\right)$ ou en polarisant le substrat.

Le problème est de savoir si ces formes c. f. c. qualifiées quelquefois "d'anormales » sont des formes allotropiques plus ou moins stables d'un élément qui se présente habituellement sous une forme différente c. c. ou hexagonale, s'il s'agit d'une modification du réseau stabilisée par une impureté introduite au moment de la condensation, ou encore s'il s'agit d'une réaction chimique avec des gaz résiduels actifs présents dans l'enceinte ou à la surface du substrat. Nous penchons plutôt pour cette dernière hypothèse.

En effet, nous avons pu montrer qu'on évite la formation d'un dépôt c. f. c. en dégazant soigneusement le substrat en le chauffant pendant un certain temps à une température supérieure à la température de dépôt, en purifiant l'atmosphère de l'enceinte par une pulvérisation préalable suffisamment prolongée et aussi en appliquant au substrat, pendant la croissance du dépôt, une polarisation négative d'une cinquantaine de volts pour désorber les gaz susceptibles de réagir. Ainsi, avec une pression d'argon aussi élevée que $5 \times 10^{-3}$ torr, à $200^{\circ}$, mais après pulvérisation préalable de 15 minutes et en polarisant le substrat sous $50 \mathrm{~V}$ on a obtenu une couche normale c. c. très bien orientée.

Mais il est difficile de préciser à quel composé s'apparente la forme c. f. c, car, d'une part, la maille c. f. c. voisine de 4,42 $\AA$ peut correspondre à différents composés du niobium et d'autre part, il n'est pas facile d'identifier avec certitude les gaz qui réagissent avant ou pendant la condensation.

N. Terao $[6,7,8,9]$, dans son étude très complète sur l'oxydation, la nitruration et la carburation des films minces de niobium par diffraction électronique signale, à plusieurs reprises, une forme c. f. c. de maille $4,40 \AA$ qu'il attribue à un oxyde $\mathrm{Nb}-\mathrm{O}$ dont le diagramme, dit-il, pourrait être facilement confondu avec celui du nitrure $\mathrm{NbN}$ ou du carbure $\mathrm{NbC}$.

Toutefois il n'obtenait ces composés qu'à des températures assez élevées $\left(>1200^{\circ} \mathrm{C}\right)$ et pensait qu'on pouvait les distinguer de $\mathrm{Nb}-\mathrm{O}$ en chauffant dans un vide assez poussé, meilleur que $10^{-4}$ torr, $\mathrm{Nb}-\mathrm{O}$ devant seul se transformer en $\mathrm{NbO}_{2}$.

Il nous a donc paru intéressant d'étudier le comportement des couches de niobium obtenues par pulvérisation triode au cours de chauffages, dans un diffractographe, jusqu'à $800^{\circ}$ environ, sous différentes pressions d'air résiduel.

Traitements thermiques dans le vide d'un diffractographe. - Nous avons utilisé pour cela l'appareil dans lequel nous avions déjà étudié avec J. J. Trillat [10] l'oxydation superficielle du niobium massif et l'oxydation de couches minces obtenues par bombardement électronique. Il s'agissait dans tous les cas d'échantillons ayant la structure normale c. c. et l'oxydation avait lieu soit dans l'air, soit dans le diffractographe, sous une pression d'air résiduel de quelques $10^{-3}$ torr, ou voisine de $10^{-5}$ torr.

Les résultats de cette première étude sont résumés sur le tableau I : 
TABlEAU I

Oxydation du niobium : étude par diffraction électronique

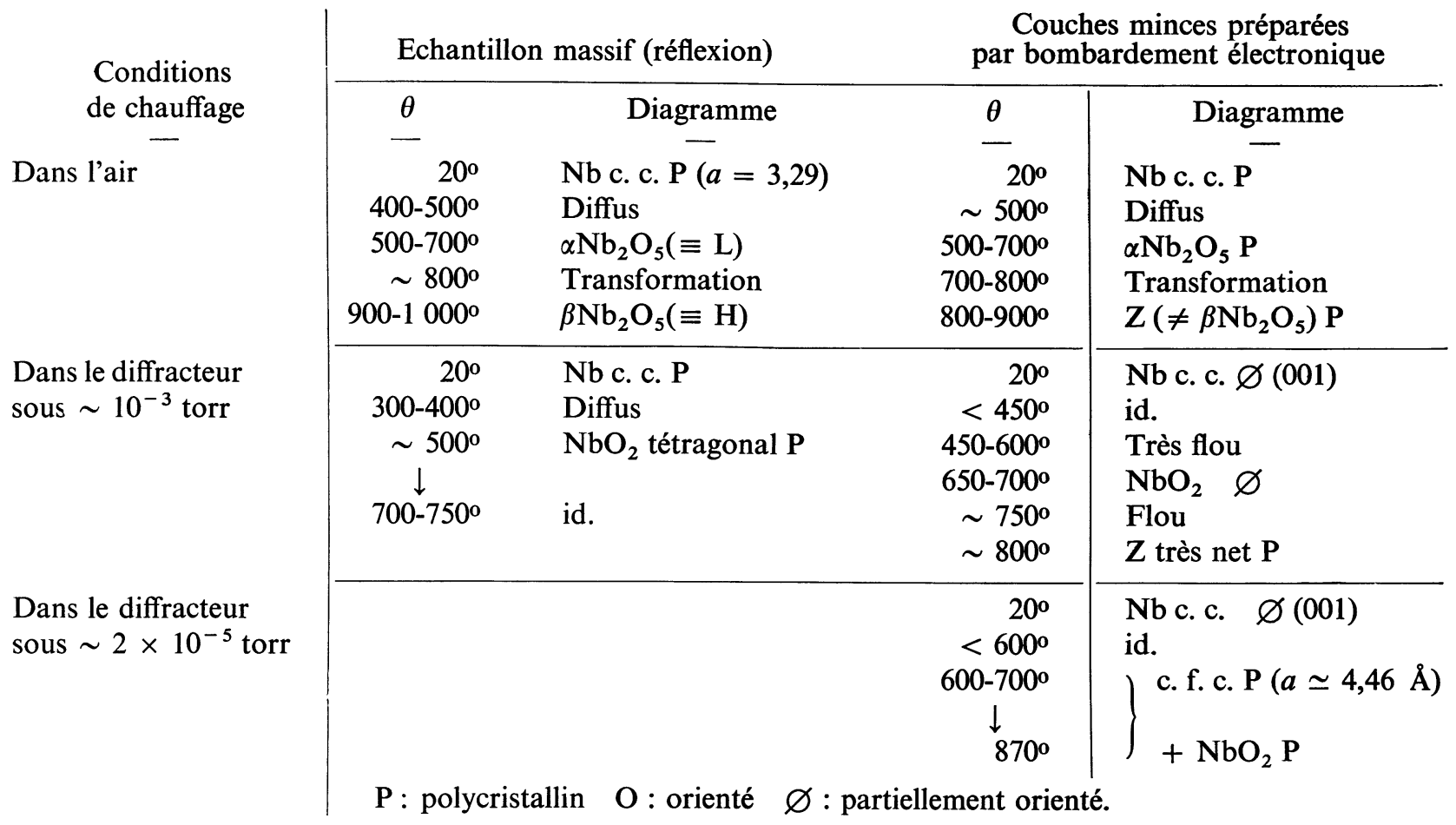

$1^{\circ}$ Dans l'air, vers $500^{\circ}$, on observe la formation $\mathrm{du}$ pentoxyde $\alpha-\mathrm{Nb}_{2} \mathrm{O}_{5}$ aussi bien en couches minces que sur le métal massif. Après une transformation aux environs de $800^{\circ}$, on peut identifier sur le métal massif la forme $\beta-\mathrm{Nb}_{2} \mathrm{O}_{5}$ alors qu'en couche mince il apparaît un diagramme différent très net (Fig. 10)

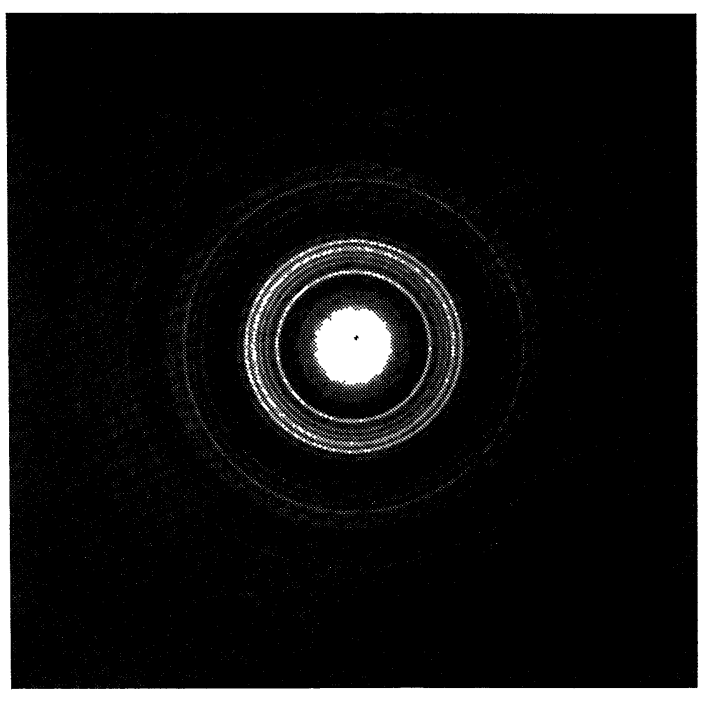

FIG. 10. - Diagramme Z.

que nous avons désigné par Z. Il n'est pas possible en effet de l'identifier à l'une des nombreuses modifications du pentoxyde décrites dans la littérature [11 à 15] mais, étant donné les conditions dans lesquelles nous l'avons plusieurs fois obtenu au cours de cette étude,

\section{TABleau II}

\section{Diagramme $\mathbf{Z}$}

Distances réticulaires et intensités relatives

\begin{tabular}{|c|c|c|c|}
\hline$I$ & $d(\AA)$ & $I$ & $d(\AA)$ \\
\hline- & - & - & - \\
\hline tf & 8,6 & $\mathrm{aF}$ & 1,97 \\
\hline a. f. & 6,2 & $\mathrm{~m}$ & 1,86 \\
\hline$f$ & 5,5 & $\mathrm{~F}$ & 1,76 \\
\hline $\mathrm{f}$ & 4,4 & $\mathrm{tf}$ & 1,72 \\
\hline $\mathrm{TF}$ & 3,94 & $\mathrm{tf}$ & 1,68 \\
\hline $\mathrm{aF}$ & 3,47 & $\mathrm{~m}$ & 1,65 \\
\hline $\mathrm{aF}$ & 3,22 & $\mathrm{~m}$ & 1,61 \\
\hline $\mathrm{aF}$ & 3,03 & $\mathrm{tf}$ & 1,54 \\
\hline $\mathrm{m}$ & 2,94 & $\mathrm{tf}$ & 1,50 \\
\hline $\mathrm{F}$ & 2,78 & $\mathrm{~m}$ & 1,45 \\
\hline $\mathrm{aF}$ & 2,60 & $\mathrm{f}$ & 1,40 \\
\hline f & 2,45 & a.f. & 1,36 \\
\hline tf & 2,28 & $\mathrm{~F}$ & 1,32 \\
\hline f & 2,14 & : & : \\
\hline $\mathrm{tf}$ & 2,08 & : & \\
\hline
\end{tabular}

nous pensons qu'il s'agit d'une forme de transition de $\mathrm{Nb}_{2} \mathrm{O}_{5}$. Les distances réticulaires et les intensités relatives qui lui correspondent sont consignées sur le tableau II.

$2^{\circ}$ Sous $10^{-3}$ torr le dioxyde $\mathrm{NbO}_{2}$, tétragonal, apparait vers $600^{\circ}$; vers $800^{\circ}$, en couche mince, on retrouve $Z$.

$3^{0}$ Sous pression très réduite $\left(10^{-5}\right.$ torr $), \mathrm{NbO}_{2}$ se 


\section{TABLEAU III}

Pulvérisation triode du niobium

Chauffage sous vide des dépôts $c . f$. c.

\begin{tabular}{|c|c|c|c|c|}
\hline \multicolumn{2}{|c|}{ Préparation } & \multirow{2}{*}{$\begin{array}{l}\mathrm{p} \text { (torr) } \\
\text { pendant le } \\
\text { chauffage }\end{array}$} & & \multirow[b]{2}{*}{ Diagramme } \\
\hline$\underline{\theta_{\mathrm{NaCl}}}$ & $\mathrm{p}_{\text {argon }}$ & & 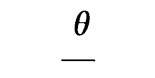 & \\
\hline $300^{\circ}$ & $10 \mu$ & $2 \times 10^{-5}$ & $\begin{array}{r}20^{\circ} \\
<700^{\circ} \\
750-780^{\circ}\end{array}$ & $\begin{array}{l}\text { c. f. c. }(a=4,42 \AA) \mathrm{O}(001) \\
\text { id. } \\
\text { id. }+\mathrm{NbO}_{2} \varnothing\end{array}$ \\
\hline $200^{\circ}$ & $6 \times 10^{-4}$ torr & $2 \times 10^{-5}$ & $\begin{array}{r}20^{\circ} \\
\text { vers } 750^{\circ}\end{array}$ & $\begin{array}{l}\text { c. f. c. } \mathrm{P}+\mathrm{O}(001)(a=4,42) \\
\text { Formation de } \mathrm{NbO}_{2}+\text { diagramme initial }\end{array}$ \\
\hline $200^{\circ}$ & $6 \times 10^{-4}$ torr & $<10^{-5}$ & $\begin{array}{r}20^{\circ} \\
780^{\circ} \\
800^{\circ}\end{array}$ & $\begin{array}{l}\text { c. f. c. } \mathrm{P}+\mathrm{O}(001) \\
\text { Formation de } \mathrm{NbO}_{2} \\
\text { Diagramme initial }+\mathrm{NbO}_{2}\end{array}$ \\
\hline $300^{\circ}$ & $10 \mu$ & $6 \times 10^{-3}$ & $\begin{array}{r}20^{\circ} \\
<500^{\circ} \\
550-600^{\circ} \\
600-750^{\circ} \\
770-790^{\circ} \\
800^{\circ}\end{array}$ & $\begin{array}{l}\text { c. f. c } \mathrm{P}+\mathrm{O}(001)(a \simeq 4,42) \\
\text { id. } \\
\text { id. }+ \text { anneau flou au centre } \\
\text { Apparition d'anneaux supplém. + taches initiales }(001) \\
\text { Diagramme } \mathrm{Z}+\text { taches légèrement déplacées }(a \simeq 4,40) \\
\mathrm{Z} \text { très net }+ \text { taches déplacées }(\rightarrow a=4,20 \AA: \mathrm{NbO} \text { ?) }\end{array}$ \\
\hline
\end{tabular}

$\mathbf{P}$ : polycristallin $\mathbf{O}$ : orienté $\varnothing$ : partiellement orienté.

forme également mais à température plus haute et nous avions observé simultanément un diagramme c. f. c. de maille voisine de 4,46 $\AA$ que nous avions attribué au carbure $\mathrm{NbC}$.

$4^{0}$ Enfin, à aucun moment, nous n'avons décelé le monoxyde $\mathrm{NbO}$, c. f. c., de maille $a=4,20 \AA$.

Des traitements thermiques analogues effectués sur les couches de niobium obtenues par pulvérisation triode ont donné les résultats suivants :

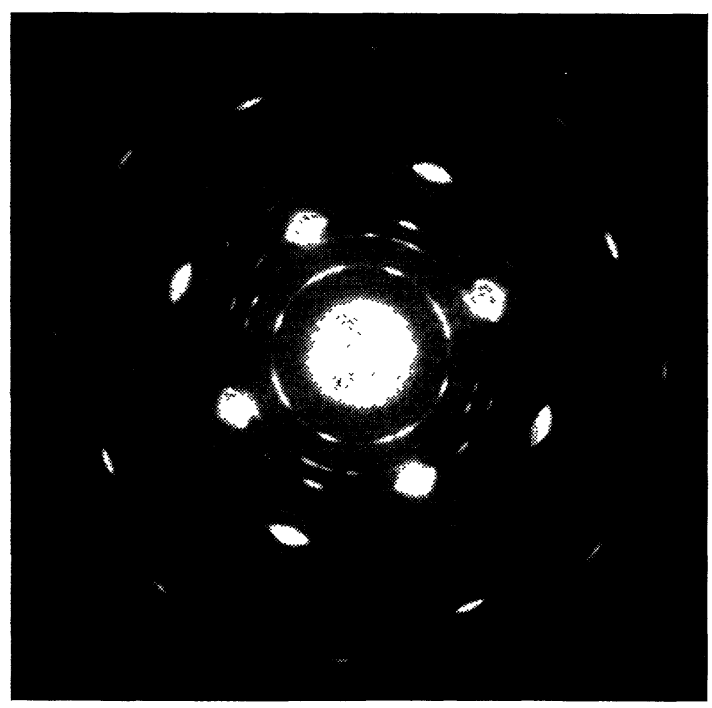

FIG. 11. $-750^{\circ}$ sous $2 \times 10^{-5}$ torr : diagramme initial c. f. c. $+\mathrm{NbO}_{2}$. a. Couches de STRuCture CubiQue a faCes CENTRÉES. - Les résultats sont rassemblés sur le tableau III :

1. Sous vide poussé, voisin de $10^{-5}$ torr, on constate la formation de $\mathrm{NbO}_{2}$, vers $750^{\circ}-800^{\circ}$, mais la transformation n'est pas totale et le diagramme initial subsiste (Fig. 11). Ainsi la forme c. f. c. se conserve en partie, très stable, sans changement de maille appréciable. On peut donc penser qu'elle contient de l'oxygène $(\mathrm{Nb}-\mathrm{O})$ mais également du nitrure ou du carbure.

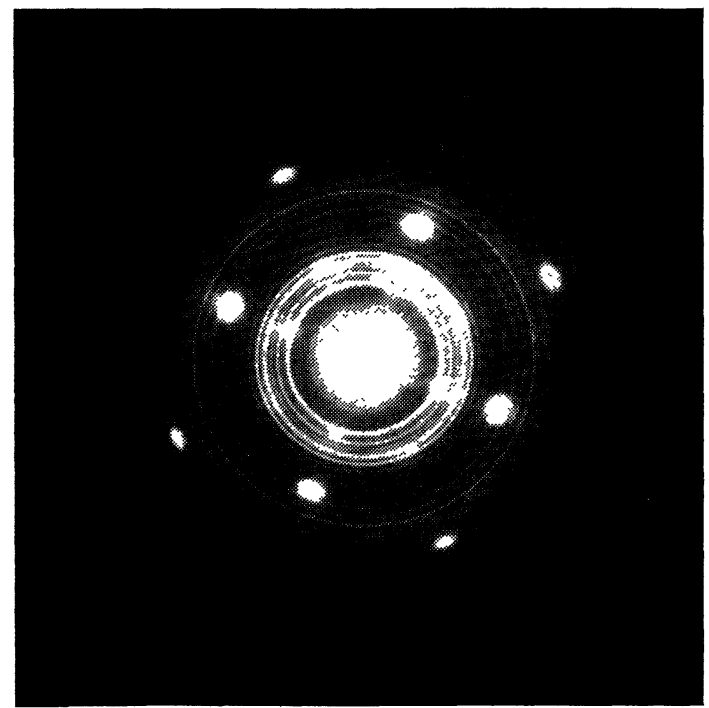

FIG. $12 .-800^{\circ}$ sous $6 \times 10^{-3}$ torr : forme c. f. c. $\rightarrow \mathrm{Z}+\mathrm{NbO}$ (?). 
2. Sous pression plus élevée $\left(6 \times 10^{-3}\right.$ torr $)$, on voit apparaitre progressivement un diagramme qui, à $800^{\circ}$, est identique au diagramme $\mathrm{Z}$ signalé plus haut, tandis que les taches (hko) du diagramme initial $(a=4,42 \AA)$ se sont déplacées et correspondent à la maille c. f. c. de 4,20 $\AA$ donnée pour NbO (Fig. 12).

$b$. Couches de STRucture Normale c. c. -1 . Sous vide poussé, $<2 \times 10^{-5}$ torr, on a observé systématiquement en chauffant les couches normales c. c. obtenues par pulvérisation triode un dédoublement des taches de l'anneau (110) entre $500^{\circ}$ et $700^{\circ}$ (Fig. 13) et la formation d'un composé c. f. c. de maille $a \simeq 4,45 \AA$.

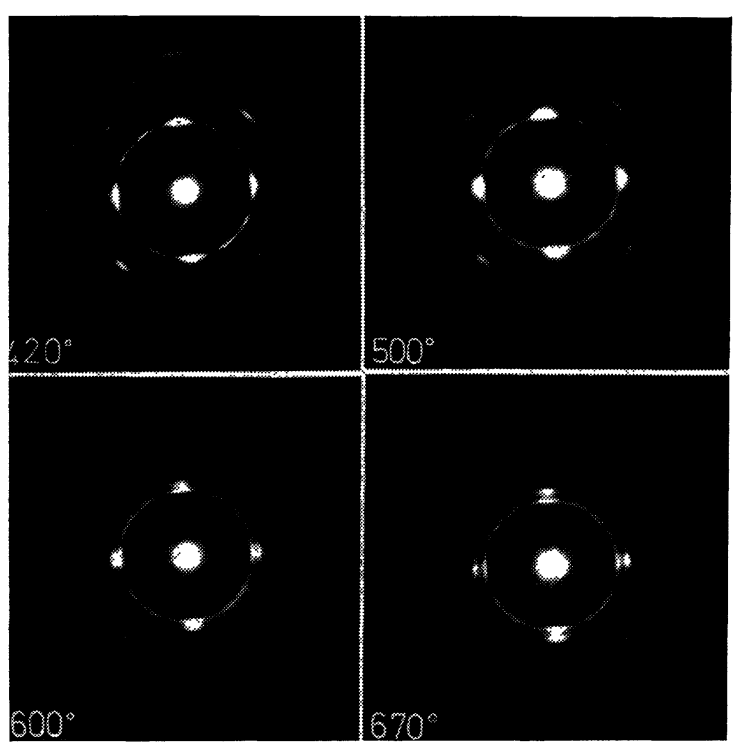

FIG. 13. $-\mathrm{Nb}$ c. c. sous $2 \times 10^{-5}$ torr. Dédoublement des taches et transformation en un réseau c. f. c $(a=4,45 \AA)$.

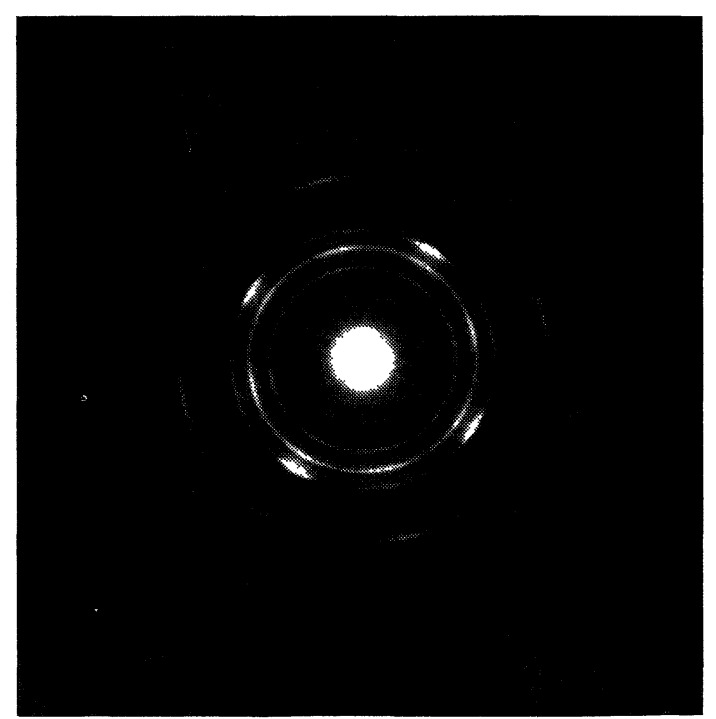

FIG. 14. $-\mathrm{Nb}$ c. c. à $750^{\circ}$ sous $2 \times 10^{-5}$ torr $\rightarrow$ diagramme non identifié (c. s. $a=4,25 \AA$ ).

Ensuite, vers $700^{\circ}-750^{\circ}$ une première transformation fait apparaître un diagramme (Fig. 14) non identifié qui correspond à un réseau cubique simple de maille $a=4,25 \AA$ auquel s'ajoutent, vers $800^{\circ}$, les raies de $\mathrm{NbO}_{2}$ (Fig. 15).

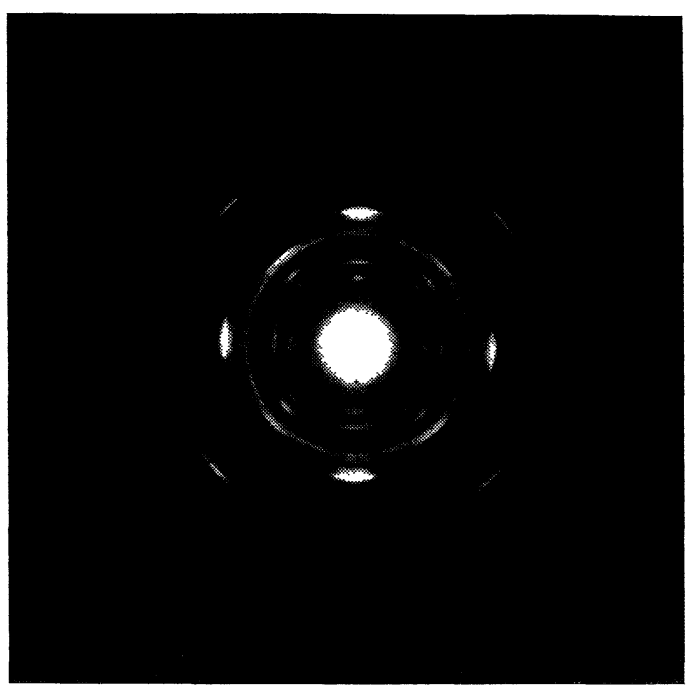

FIG. 15. - Nb c. c. à $800^{\circ}$ sous $2 \times 10^{-5}$ torr $\rightarrow$ diagramme (14). $+\mathrm{NbO}_{2}+$ c. f. c. (?).

2. Sous vide moins poussé $\left(7 \times 10^{-3}\right.$ torr $)$ on retrouve vers $800^{\circ}$ le diagramme $Z$ déjà décrit après une série de transformations assez complexes : on note en effet vers $450^{\circ}$ un dédoublement des taches comme précédemment mais suivi très rapidement par l'apparition de nombreux anneaux correspondant à la formation de $\mathrm{NbO}_{2}$ puis de $\mathrm{Nb}_{2} \mathrm{O}_{5}$ avec des diagrammes intermédiaires souvent diffus.

Cette forme c. f. c. qui apparaît dans le diffractographe dès $450^{\circ}$ atteint progressivement une maille très voisine de celle qui se forme dans l'enceinte de pulvérisation cathodique si l'on ne prend pas de précautions particulières pour l'éviter. Les mêmes composés interstitiels sont susceptibles de se former surtout avec l'oxygène et le carbone et il est intéressant de suivre progressivement la transformation du réseau.

Conclusion. - L'ensemble de ces observations ne permet pas de conclure quant à la composition de la

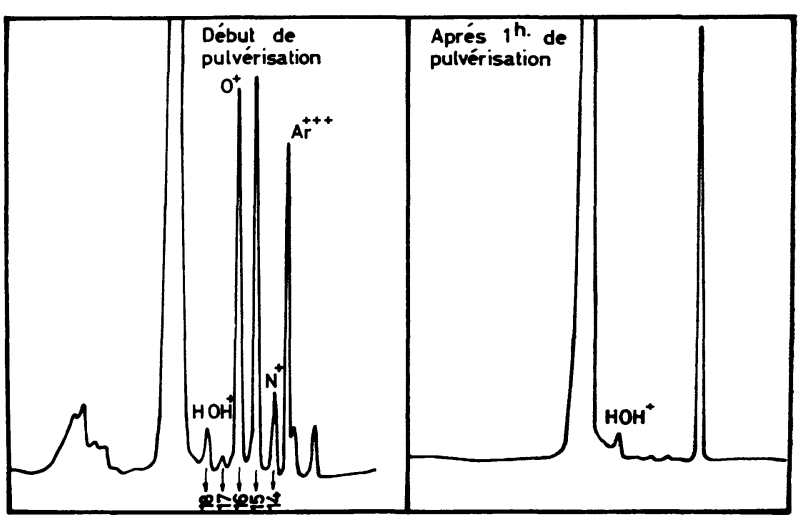

FIG. 16. - Evolution de la composition de l'atmosphère résiduelle au cours de la pulvérisation. 
forme cubique à faces centrées. Pour le moment nous pensons qu'elle est due en partie à l'introduction d'un peu d'oxygène dans le réseau du niobium. Cet oxygène peut en effet provenir de l'atmosphère résiduelle et éventuellement de la décomposition de traces de vapeur d'eau. On peut constater, en suivant l'évolution de la composition de l'atmosphère résiduelle de l'enceinte (Fig. 16) que l'azote, l'oxygène et les traces d'hydrocarbures disparaissent rapidement ce qui permet de penser que ces gaz réagissent avec le métal dans les premiers stades de la pulvérisation. En tra- vaillant dans un vide initial plus propre et plus poussé on n'observe pas la forme c. f. c.

Il serait intéressant de pouvoir identifier et doser avec précision les gaz réellement fixés dans les couches à structure anormale, de façon à savoir si cette structure correspond ou non à un composé défini. Les essais effectués dans ce sens par Chopra [3] et Sosniak [5] montrent que les concentrations en gaz $\left(\mathrm{N}_{2}, \mathrm{O}_{2}\right.$, etc.) effectivement fixées sont très faibles et semblent insuffisantes pour permettre la formation d'un composé stœchiométrique.

\section{Bibliographie}

[1] Sella (C.) et Coppens (R.), C. R. Acad. Sci., 1967, $265,410$.

[2] Miska (E.) et Gillet (M.), C. R. Acad. Sci., 1967, 264, 1267.

[3] Chopra (K. L.), Randlett (M. R.) and Duff (R. H.), Phil. Mag., 1967, 16, 261.

[4] Hutchinson (T. E.) and Olsen (K. H.), J. Appl. Phys., 1967, 38, 4933.

[5] Sosniak (J.), J. Appl. Phys., 1968, 39, 4157.

[6] Terao (N.), Japan. J. Appl. Phys., 1963, 2, 156.

[7] Terao (N.), Japan. J. Appl. Phys., 1964, 3, 104.
[8] Terao (N.), Japan. J. Appl. Phys., 1965, 4, 8.

[9] Terao (N.), Japan. J. Appl. Phys., 1965, 4, 353.

[10] Trillat (J. J.) et Tertian (L.), Mém. Sc. Metallurg., 1963, LX, 23.

[11] Brauer (G.), Z. Anorg. Chem., 1941, 248, 1.

[12] Frevel (L. K.) et RinN (H. W.), Analyt. Chem., 1955, $27,1329$.

[13] SHÄFer (M. W.) et Roy (R.), Z. Krist., 1958, 110, 241.

[14] GoldSMith (H. J.), J. Inst. Metals, 1959, 87- 235.

[15] Schäfer (H.), Gruehn (R.) and Schulte (F.), Angew. Chem. Internat. Edit., 1966, 5, 1. 